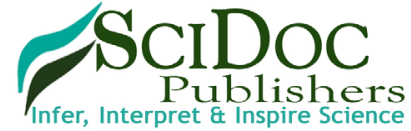

\section{Anemia Associated with Autism Spectrum Disorder}

Research Article

Castro $\mathrm{K}^{1,2^{*}}$, Marchezan $\mathrm{J}^{1,3}$, Faccioli $\mathrm{LS}^{2}$, Riesgo $\mathrm{R}^{1,3}$, Perry IS $\mathrm{IS}^{2,4}$

${ }^{1}$ Programa de Pós Graduação em Saúde da Criança e do Adolescente, Universidade Federal do Rio Grande do Sul, Porto Alegre, RS, Brasil.

${ }^{2}$ Centro de Estudos em Alimentação e Nutrição (CESAN), Hospital (PPGSCA) de Clínicas de Porto Alegre- Universidade federal do Rio Grande do Sul, Porto Alegre, RS, Brasil.

${ }^{3}$ Unidade de Neuropediatria, Hospital de Clínicas de Porto Alegre, Universidade Federal do Rio Grande do Sul, Porto Alegre, RS, Brasil.

${ }^{4}$ Programa de Pós Graduação em Saúde Coletiva, Universidade do Extremo Sul Catarinense, Criciúma, SC, Brasil.

\title{
Abstract
}

This study aims to investigate the prevalence of anemia in children and adolescents with autism, between 3 and 18 years old, through a retrospective review in medical records. Additionally, items relating to food selectivity and/or restriction were assed. A total of 118 medical reports were reviewed concerning hematimetric parameters (serum ferritin, iron, hemoglobin, hematocrit, mean corpuscular volume, and red cell distribution width). We found that $28.07 \%$ had anemia based on hemoglobin, according to the WHO; in these patients, $21.5 \%$ had some type of food selectivity. Regarding anemia as a result of iron deficiency (ferritin below $12 \mu \mathrm{g} / \mathrm{l}$ for children under five years and below $15 \mu / \mathrm{L}$ for over five years) we verified that 19 patients presented a positive result. The high frequency of adolescents and children with anemia suggests that these parameters should be measured as part of a routine investigation in Autism Spectrum Disorder as a standard practice.

Keywords: Autistic Disorder; Hematological Parameters; Anemia; Iron Deficiency Anemia.

\section{Introduction}

Autism Spectrum Disorder (ASD) is a neurodevelopmental disorder characterized by persistent deficits in social communication and interaction, and the presence of repetitive behaviors and/or restricted interests $[1,2]$. The heterogeneity of etiology is without doubt a major obstacle, because an overlap of symptoms masks the actual path to a real cause of ASD [1]. Despite the increasing frequency of diagnosis and numerous suggestions and/or theories as to the origins of this apparent sudden epidemic of ASD, a unique cause has not been identified [3]. ASD affects one in every 68 children in the United States, and prevalence is increasing [4].

Anemia diminishes oxygen transportation in the body, resulting in potentially irreversible growth and developmental consequences for children. This disease is a condition resulting from insufficient healthy red blood cells to carry an adequate amount of oxygen to tissues. Anemia symptoms vary depending on the cause: fatigue, weakness, pale skin, a fast or irregular heartbeat, shortness of breath, chest pain, dizziness, cognitive problems, cold hands and feet, headache. Initially, anemia can be so mild as to go unnoticed, but its symptoms increase as it progresses [5, 6]. Globally, 43\% of young children (6-59 months) [7] and 25\% of older children (5-15 years) [6], a combined 600 million, are estimated to be anemic.

Iron is a crucial micronutrient in early neurodevelopment. In the brain, iron contributes to neurotransmitter production, myelination, and immune function [8]. There is a considerably an important role of iron in cognitive, behavioral and motor development [8], and iron deficiency early in life can affect these parameters [9]. Long term, iron deficiency can cause neurocognitive problems, such as psychomotor development delay [10]. The current parameters used for the diagnosis of iron deficiency include serum ferritin and iron (transferrin) saturation [11].

Anemia due to iron deficiency is the most common nutritional deficiency in many developing countries, causing major health, social and economic consequences [12]. Some studies reported

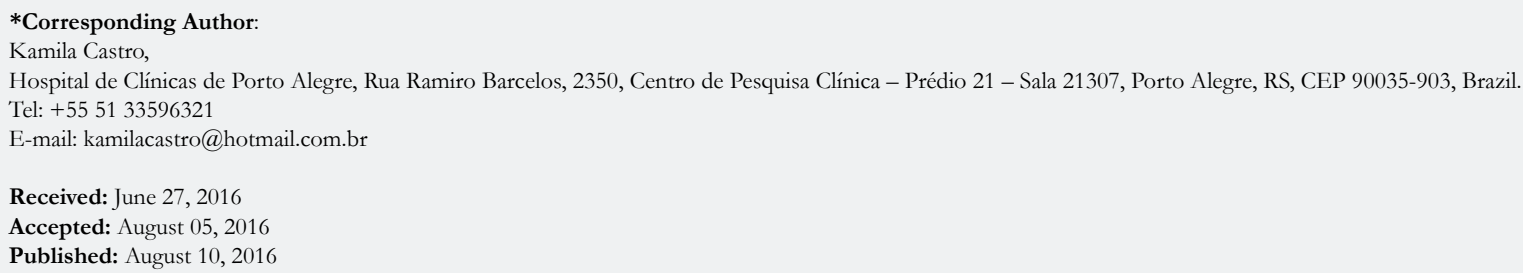

Copyright: Castro $\mathbf{K}^{\circ}$ 2016. This is an open-access article distributed under the terms of the Creative Commons Attribution License, which permits unrestricted use, distribution and reproduction in any medium, provided the original author and source are credited. 
lower ferritin levels in different neurological diseases such as attention deficit hyperactivity [13] and Tourette's syndrome [14]. Children and adolescents with ASD may have deficits of iron levels increasing the prevalence of anemia in this population [1519]. In addition, the high frequency of low iron intake in these children is thought to be associated with feeding difficulties and food selectivity [20]. Thus, the aim of this study was to review hematimetric data from children and adolescents with ASD, outpatients in a Hospital in Porto Alegre, Brazil.

\section{Methods}

\section{Participants}

Participants were selected from an ASD specialized Neuropediatric Ambulatory in a Clinical Hospital in Porto Alegre and a retrospective review was conducted using medical records from December 2012 to July 2015. The inclusion criteria were patients with ASD diagnosis by a pediatric neurologist; using both genders and an age range between three to eighteen years.

\section{Clinical and Hematological parameters}

The clinical parameters noted were age of onset of symptoms, age of diagnosis, and use of medication (s). To assess the presence of food selectivity or restriction that could play an etiological role in anemia, this information was collected in the medical records in the same period as the blood tests. Food selectivity can be defined in the literature with multiple terms referring to a range of concepts including food refusal, a limited repertoire of accepted foods, and high-frequency single food intake [20]. In our study, we define food selectivity as a small variety or color of food, limited texture, and restriction with eating schedules or accessories to eating. Hemoglobin, hematocrit, red and white blood cells count and mean corpuscular volume (MCV) were also obtained from medical records and analyzed through the parameters in the literature, stratified by age and gender [21]. Patients with ferritin below $12 \mu \mathrm{g} / \mathrm{L}$ for children under five years and below $15 \mu \mathrm{g} / \mathrm{L}$ for over five years were considered to have anemia [21]. As iron deficiency anemia is the main microcytic anemia in childhood, the presence of microcytosis was also considered iron deficiency anemia, in the absence of ferritin data.

\section{Statistical analysis}

Descriptive analysis was performed using SPSS version 22.0. The results are shown with mean \pm standard deviation or median (minimum - maximum).

\section{Ethics Aspects}

The Ethics Committee at the Clinical Hospital of Porto Alegre approved this study for all aspects (protocol number 14-0674).

\section{Results}

During the period, 203 medical records of patients with ASD were analyzed. A total of 118 medical records with blood exams were reviewed. With a mean age of $10.7 \pm 4.5$ years, approximately $79 \%$ of these patients were male. The mean age of onset of symptoms was $2.53 \pm 1.08$ and the age of diagnosis of the patients was 5.5 \pm 2.7 years. Other clinical and demographic characteristics are described in Table 1.

Hematological parameters are shown in Table 2. Considering the total number of outpatients, the prevalence of anemia was $28.07 \%$. For anemia characterized through iron deficiency, eleven patients showed lower levels of ferritin and eight patients showed lower MCV, accounting for $33.3 \%$ of total anemia (Figure 1).

A total of 13 girls and 44 boys had anemia. Considering preschool children aged less than six years old (median: 4.6; 3.41-6.00), there were 10/21 patients with anemia, while in older children and adolescents (median: 12.76; 6.25-18.58 years old), 47/97 showed this disorder.

Iron deficiency anemia was present in $9.35 \%(n=19)$ of the total number of outpatients, in 14 ASD boys, and in 5 ASD girls. The mean age of these boys and girls was 11.76 and 12.71 years old respectively.

Table 1. Demographic and clinical characteristics of Autism Spectrum Disorder pediatric outpatients.

\begin{tabular}{|c|c|}
\hline Variable & N (\%) \\
\hline Gender & \\
Female & $24(20.3)$ \\
Male & $94(79.7)$ \\
\hline Epilepsy & \\
Yes & $32(27.1)$ \\
No & $86(72.8)$ \\
\hline Drugs & $104(88.1)$ \\
Yes & $14(11.9)$ \\
No & \\
\hline Food selectivity + & $29(25.6)$ \\
Yes & $84(74.3)$ \\
No &
\end{tabular}

+A small variety or color of food, limited texture, and restriction with schedules to eat or accessories to do the meals reported in medical records. $\mathrm{n}=118$ medical records of patients with blood exams. 
Table 2. Hematological parameters of Autism Spectrum Disorder pediatric outpatients.

\begin{tabular}{|c|c|}
\hline Variable & Results \\
\hline Ferritin $(\mathrm{ng} / \mathrm{mL})^{*}$ & $30.60 \pm 10.94(8.1-165.3)$ \\
\hline Iron $(\mu \mathrm{g} / \mathrm{dL})^{*}$ & $95.00 \pm 37.65(9.1-203)$ \\
\hline Hemoglobin $(\mathrm{ug} / \mathrm{dL})$ & $12.86 \pm 1.17(7.9-20.3)$ \\
\hline Hematocrit $(\%)$ & $37.60 \pm 3.11(21.8-40.2)$ \\
\hline MCV $(\mathrm{fL})$ & $82.91 \pm 5.21(65.8-95.2)$ \\
\hline RDW $(\%)$ & $13.27 \pm 0.88(11.7-16.6)$ \\
\hline
\end{tabular}

MCV: Mean Corpuscular Volume; RDW: Red Cell Distribution Width. *The analysis to ferritin and iron levels $\mathrm{n}=80$ patients for the other parameters analyzed $\mathrm{n}=118$. The reference values are according to WHO. Iron Deficiency Anaemia Assessment, Prevention, and Control A guide for programme managers. 2001.[21]

Figure 1. Anemia inoutpatients with Austin Spectrum Disorder (ASD).

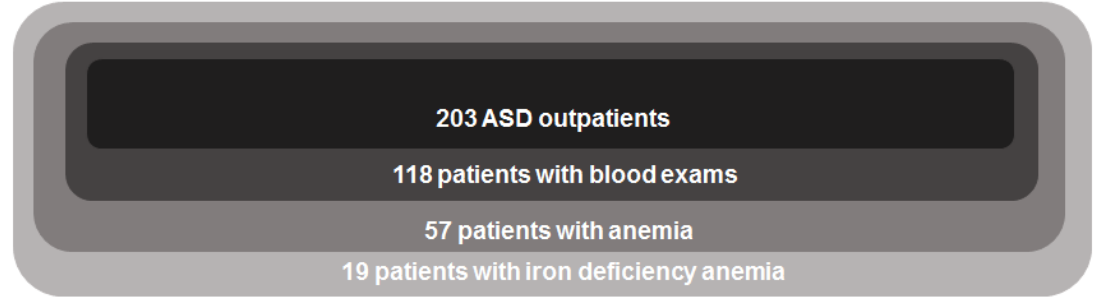

A total of 29 patients had some type of food selectivity, but when the anemic patients were analyzed ( $\mathrm{n}=57), 21.5 \%$ showed this behavior. In addition, for patients with iron deficiency $15.78 \%$ had eating restrictions.

\section{Discussion}

Our study reported a prevalence of $28.07 \%$ of anemia in patients with ASD. Other studies have also showed iron deficiency and anemia in this neurological disorder. However, the studies vary according to different parameters of the analyses, e.g., transferrin levels, iron levels or hematimetric levels in global form [15-19]. National population data indicate that the prevalence of anemia in children under five years of age is $20.9 \%$, and in children under two years is $24.1 \%$. Specifically on our region, South Brazil, 605 children were assessed and the prevalence of anemia was close to national data, at $21.5 \%$ [22].

In studies with autistic children and non-autistic children that compared hematimetric levels, transferrin levels were lower [19], and intraerythrocyte and plasma non-protein-bound iron levels were significantly increased in autistic patients [23].

Anemia has been reported in the literature in $3 \%$ to $15.5 \%$ of children with ASD $[15,17,24-26]$. A recent study reinforced that iron deficiency and iron deficiency anemia were more common in children with global developmental delay and/or ASD than in the general population [24]. It's noteworthy that both data sets, in ASD children as well in the general population [24], evidenced a lower prevalence than in our study.

When stratified for age, although not statistically significant, iron deficiency was more prevalent in preschool-aged than school-aged children $(32.4 \%$ vs. $20.3 \%)$ and anemia was higher in older cases $(13.5 \%$ vs. $16.5 \%)$, respectively [15]. In our study, we found similar rates of anemia in preschool and school aged patients. Considering that our younger sample is very small, more investigation is needed.

A low intake of iron by these children is also reported [27] and could be related to anemia prevalence. However, considering studies that evaluated food records, there is controversy. One study showed fewer than $2 \%$ of ASD children had iron intake below the estimated average requirement. In the same study, $8 \%$ had serum ferritin lower than $12 \mu \mathrm{g} / \mathrm{L}, 1 \%$ had iron deficiency defined by both low serum ferritin and serum transferrin, and just one subject had iron deficiency anemia [17]. On the other hand, the iron intake compared to the RDIs showed a 5.24 fold higher risk of failing to meet recommendation in ASD children compared to controls [28]. In addition, 35\% of school-age children with ASD had insufficient iron intake [16], and an inadequate consumption of this micronutrient was more frequent in children with ASD compared to controls $[27,29]$ or to reference values $[27,30,31]$.

A low iron intake in these children could be associated with feeding difficulties and food selectivity [20], however, in our sample with anemia, just $21.5 \%$ had some food selectivity register and only $15.78 \%$ of those with iron deficiency anemia showed this behavior. According to Hergrüner et al., (2012) [15], impaired iron absorption could be responsible for this effect. However, the hypothesis is contradicted by an intervention study from Dossman et al., (2007) [16] in which iron supplementation restores ferritin and iron levels, weakening this probability.

In the present study, the frequency of children and adolescents with anemia was classified in the moderate category of public 
health significance, on the basis of prevalence estimated from blood levels of hemoglobin and hematocrit, according to WHO criteria [21]. This result justified redoubling prevention efforts, considering the deleterious effects on the general cognitive status in the long and short term. Furthermore, the described restrictions on feeding behavior, suction problems, chewing or swallowing difficulties, adequacy of food intake and possible macro and micronutrient deficiencies, as well as some types of chronic medication may be related to the risk of these patients to develop anemia. The superimposing factors that surround anemia, suggest that these parameters should be measured as part of routine investigation.

The limitations of the present study must be considered, such as a local clinical sample size, lack of a control group, and the wide range of patients without available blood exams. In addition, a food survey was not employed. Further studies with larger samples and control groups would help to determine out if there is indeed a higher prevalence of anemia in these children, as well as assess the adequacy of their inadequate iron intake.

\section{Acknowledgements}

This study was financed by the Brazilian National Council for Scientific and Technological Development (CNPq), by the Rio Grande do Sul Research Foundation (FAPERGS), and by the Hospital de Clínicas de Porto Alegre Research and Event Incentive Fund (FIPE).

\section{References}

[1]. Happé F, Ronald A (2008) The 'fractionable autism triad'. a review of evidence from behavioural, genetic, cognitive and neural research. Neuropsychol Rev.18(4):287-304.

[2]. Diagnostic and Statistical Manual of Mental Disorders (2013) (DSM-V). $5^{\text {th }}$ ed: American Psychiatric Association.

[3]. Duchan E, Patel DR (2012) Epidemiology of autism spectrum disorders. Pediatr Clin North Am.59(1):27-43.

[4]. Center for Disease Control and Prevention (CDC) (2014). Prevalence of autism spectrum disorder among children aged 8 years - autism and developmental disabilities monitoring network, 11 sites, United States, 2010. MMWR Surveill Summ.63(2):1-21.

[5]. Iannotti LL, Delnatus JR, Odom AR, et al., (2015) Determinants of Anemia and Hemoglobin Concentration in Haitian School-Aged Children. Am J Trop Med Hyg. 93(5):1092-8.

[6]. McLean E, Cogswell M, Egli I, Wojdyla D, de Benoist B (2009) Worldwide prevalence of anaemia, WHO Vitamin and Mineral Nutrition Information System, 1993-2005. Public Health Nutr.12(4):444-454.

[7]. Stevens GA, Finucane MM, De-Regil LM, et al., (2013) Global, regional, and national trends in haemoglobin concentration and prevalence of total and severe anaemia in children and pregnant and non-pregnant women for 1995-2011: a systematic analysis of population-representative data. Lancet Glob Health.1(1):e16-25.

[8]. Beard JL. (2001) Iron biology in immune function, muscle metabolism and neuronal functioning. J Nutr.131(2S-2):568S-579S; discussion 580 S.

[9]. Siddappa AM, Georgieff MK, Wewerka S, Worwa C, Nelson CA, Deregnier RA. (2004) Iron deficiency alters auditory recognition memory in newborn infants of diabetic mothers. Pediatr Res.55(6):1034-1041.

[10]. Falkingham M, Abdelhamid A, Curtis P, Fairweather-Tait S, Dye L, Hooper L (2010) The effects of oral iron supplementation on cognition in older children and adults: a systematic review and meta-analysis. Nutr J. $10.1186 / 1475-2891-9-4$

[11]. Grant CC, Wall CR, Brewster D, et al. (2007) Policy statement on iron deficiency in pre-school-aged children. J Paediatr Child Health.43(7-8):513-521.

[12]. Desalegn A, Mossie A, Gedefaw L (2014) Nutritional iron deficiency anemia: magnitude and its predictors among school age children, southwest Ethiopia: a community based cross-sectional study. PLoS One. 9(12):e114059.

[13]. Konofal E, Cortese S. (2007) Lead and neuroprotection by iron in ADHD. Environ Health Perspect.115(8):A398-399.

[14]. Gorman DA, Zhu H, Anderson GM, Davies M, Peterson BS (2006) Ferritin levels and their association with regional brain volumes in Tourette's syndrome. Am J Psychiatry.163(7):1264-1272.

[15]. Hergüner S, Keleşoğlu FM, Tanıdır C, Cöpür M. (2012) Ferritin and iron levels in children with autistic disorder. Eur J Pediatr.171(1):143-146.

[16]. Dosman CF, Brian JA, Drmic IE, et al. (2007)Children with autism: effect of iron supplementation on sleep and ferritin. Pediatr Neurol.36(3):152-158.

[17]. Reynolds A, Krebs NF, Stewart PA, et al. (2012) Iron status in children with autism spectrum disorder. Pediatrics.130 (2):S154-159.

[18]. Christensen D, Van Naarden Braun K, Doernberg NS, et al. (2014) Prevalence of cerebral palsy, co-occurring autism spectrum disorders, and motor functioning - Autism and Developmental Disabilities Monitoring Network, USA, 2008. Dev Med Child Neurol.56(1):59-65.

[19]. Chauhan A, Chauhan V, Brown WT, Cohen I. (2004) Oxidative stress in autism: increased lipid peroxidation and reduced serum levels of ceruloplasmin and transferrin--the antioxidant proteins. Life Sci.75(21):2539-2549.

[20]. Marí-Bauset S, Zazpe I, Mari-Sanchis A, Llopis-González A, MoralesSuárez-Varela M (2014) Food selectivity in autism spectrum disorders: a systematic review. J Child Neurol.29(11):1554-1561.

[21]. WHO (2001) Iron Deficiency Anaemia Assessment, Prevention, and Control A guide for programme managers.

[22]. National Survey on Demography and Health of Women and Children (2006) - PNDS: dimensions of reproduction and child health.

[23]. Pecorelli A, Leoncini S, De Felice C, et al., (2013) Non-protein-bound iron and 4-hydroxynonenal protein adducts in classic autism. Brain Dev.35(2):146-154.

[24]. Sidrak S, Yoong T, Woolfenden S. (2014) Iron deficiency in children with global developmental delay and autism spectrum disorder. J Paediatr Child Health.50(5):356-361.

[25]. Latif A, Heinz P, Cook R. (2002) Iron deficiency in autism and Asperger syndrome. Autism.6(1):103-114.

[26]. Uslub ABGTFAGK. (2010) Iron deficiency in preschool children with autistic spectrum disorders. Research in Autism Spectrum Disorders.4:639-644.

[27]. Herndon AC, DiGuiseppi C, Johnson SL, Leiferman J, Reynolds A. (2009) Does nutritional intake differ between children with autism spectrum disorders and children with typical development? J Autism Dev Disord. 39(2):212-222.

[28]. Marí-Bauset S, Llopis-González A, Zazpe-García I, Marí-Sanchis A, MoralesSuárez-Varela M (2015) Nutritional status of children with autism spectrum disorders (ASDs): a case-control study. J Autism Dev Disord.45(1):203-212.

[29]. Sacco (2008) Eating habits and dietary status in young children with autism. Journal of Developmental and Physical Disabilities.20(5):437-448.

[30]. Xia W, Zhou Y, Sun C, Wang J, Wu L (2010) A preliminary study on nutritional status and intake in Chinese children with autism. Eur J Pediatr.169(10):1201-1206.

[31]. Cornish E (2008) A balanced approach towards healthy eating in autism. Journal of Human Nutrition and Dietetics. 11(6): 501-509. 